\title{
Area angles monitor area stress by responding to line outages
}

\author{
Atena Darvishi, Ian Dobson, Iowa State University \\ Akihiro Oi, Chikashi Nakazawa, Fuji Electric Co. Ltd.
}

\begin{abstract}
Area angles are a way to quantify the stress across an area of a power system by combining synchrophasor measurements of angles at the border buses of the area. One use of the area angle is to quickly monitor stress changes due to line outages within the area. We explain the area angle, illustrate its use on a 30-bus Japanese test system, and discuss how to choose areas.
\end{abstract}

\section{General NOMENCLATURE}

$\theta \quad$ bus voltage angles

$\theta_{\text {area }}$ area voltage angle

$B \quad$ susceptance matrix

$b_{\text {area }}$ area susceptance

$P \quad$ real power

$\Lambda \quad$ diagonal matrix of line susceptances

$A$ bus line incidence matrix

$\rho \quad$ power transfer distribution factor

\section{INTRODUCTION}

Synchrophasor measurements provide fast monitoring of bus voltages over a wide area. As more synchrophasors are deployed, one of the challenges is summarizing and understanding the new data. One advantageous approach is to use physical principles to combine together synchrophasor measurements into quantities that are more usable. In this paper we study how voltage angles may be combined into angles across areas of the power system. The concept of the voltage angle across a power system area is new and is described in detail in [1], [4], including how it derives from circuit theory principles. We begin with a brief overview of the voltage angle across an area in the DC load flow case. The complex voltage difference across an area in the AC load flow case is explained in [1].

The voltage phasor angle across an area is formed by suitably combining voltage angles at all the buses on the border of the area to give a single number that is the angle across the area. For example, to get the angle difference northsouth across an area, a weighted combination of angles at buses on the southern tie lines is subtracted from a weighted combination of angles at buses on the northern tie lines. The angle across an area is useful because it summarizes the circuit behavior of the area. The angle across an area behaves similarly to the angle difference across a transmission line. In particular, the angle across the area satisfies the basic circuit laws so that the effective power flow through the area is the product of the angle across the area and the effective susceptance of the area. The area angle concept is

I. Dobson (email dobson@iastate.edu) and A. Darvishi are with ECpE dept., Iowa State University, Ames IA 50011. A. Oi and C. Nakazawa are with Fuji Electric Co. Ltd., Tokyo Japan. (C)2013 IEEE a generalization of the angle across a cutset area concept developed and proposed for stress monitoring in [2], [3], [8]. (The cutset area must be chosen to extend all the way across the power system whereas the area can, in principle, be any connected area.)

The angle across an area and its special case of the cutset area angle are promising for power system monitoring, and here we are most interested in further developing its application to quantify stress across an area that is caused by line outages inside the area.

\section{A. Simple Example of Measuring Stress with an Angle.}

The motivation for using area angles to measure stress can be illustrated with the simple example of a double line joining bus $a$ to bus $b$ shown in Fig. 1 .

We assume lossless lines and a DC load flow and can compare two stress indices, the real power $P_{a b}$ flowing from $a$ to $b$ and the angle $\theta_{a b}$ between bus $a$ and bus $b$. The DC load flow equation from Ohm's law is $P_{a b}=b_{a b} \theta_{a b}$, where $b_{a b}$ is the total susceptance of the lines between $a$ and $b$. If we regard the double line as an area and the buses $a$ and $b$ as the border buses of the area, then in this simple case $\theta_{a b}$ is the area angle and $b_{a b}$ is the area susceptance.

Under normal conditions, $P_{a b}$ and $\theta_{a b}$ are proportional and both indices indicate the stress on the lines. But the indices behave very differently if one of the lines outages as illustrated in Fig. 1. The power flow $P_{a b}$ from bus $a$ to bus $b$ is unchanged, but the admittance $b_{a b}$ is halved and the angle $\theta_{a b}$ doubles. Thus the angle $\theta_{a b}$ reacts to and indicates the increase in stress caused by the outage, whereas the power flow $P_{a b}$ from bus $a$ to bus $b$ does not change and does not indicate the increase in stress.

We can also consider the limits on the indices that are determined by the thermal limits (or other flow limits) of the lines. The line outage causes the maximum power flow $P_{a b}^{\max }$ from bus $a$ to bus $b$ to halve, but the maximum angle $\theta_{a b}^{\max }$ remains the same.

In summary, the $\theta_{a b}$ index of stress is better than the power flow $P_{a b}$ index of stress because it responds to a line outage, but its maximum value remains constant. One objective of the area angle is to try to get approximately similar benefits for a bulk measurement across an entire area.

\section{B. Requirements for areas and their angles}

There are some restrictions on the allowable areas and which synchrophasor measurements are needed in order to define an area angle [1]:

1) The area must be connected. In other words, when all the tie lines of the area are tripped, the area must form only a single island. 


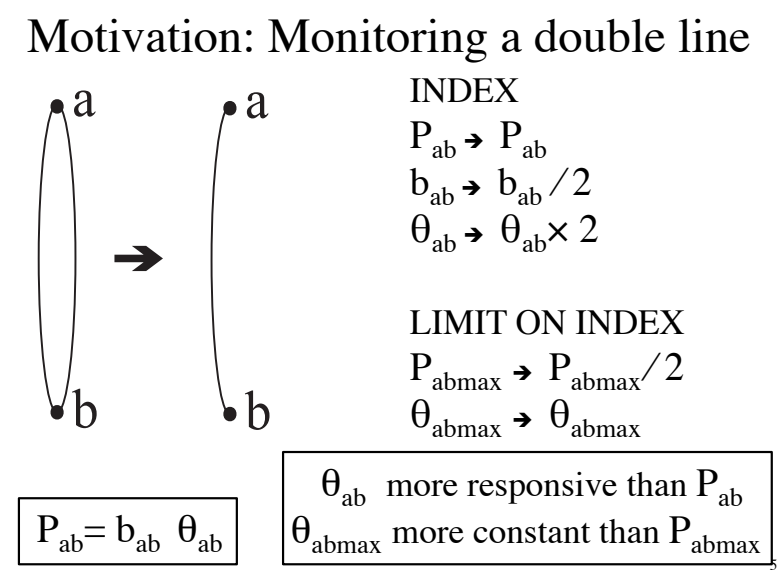

Fig. 1. Comparing $P_{a b}$ and $\theta_{a b}$ for monitoring stress for an outage in a simple double line example.

2) Synchrophasor measurements must be available at all the border buses of the area. We denote the border buses of the area by $M$. (Expressed in terms of network theory, the buses in $M$ must form a nodal cutset, so that when the buses in $M$ are removed the network is divided into two or more islands.) Each border bus corresponds to tie lines of the area to the rest of the system. This requirement prevents power flows entering or leaving the area without being tracked by the area angle. (It may be possible to relax this requirement in practice for border buses with high impedance, low voltage tie lines.)

3) Each border bus in $M$ must be classified as either an " $a$ " bus or a " $b$ " bus. We write $M=M_{a} \cup M_{b}$. Then the area angle is defined across the area from the $M_{a}$ buses to the $M_{b}$ buses. For example, $M_{a}$ can be the buses on the north border of the area and $M_{b}$ can be the buses on the south border of the area, so that the area angle is defined from north to south. Given an area with border buses $M$, there are multiple ways to choose $M_{a}$ and $M_{b}$ and each choice gives a different area angle.

4) The weights used to calculate the area angle from the border bus angles are computed from a DC load flow model of the area. A recent base case of the DC load flow model is generally available [5]. In our calculations, we use the base case DC load flow for the area angle weights, and do not, impractically, attempt to update the DC load flow model based on the outage we are trying to monitor.

It is not enough to choose an area and define a valid area angle according to these requirements; it is also important to choose an area angle that is meaningful and useful for power systems operation. In this paper, we choose an area of the transmission system between major generation and major load to try to describe with the area angle the stress resulting from the transfer of power through the area and how the stress varies with line outages inside the area.
We note that synchrophasor measurements around the border of an area can be advantageous for other applications such as locating line outages in the area [6]. (In particular, the measurements at the border can be augmented with synchrophasor measurements inside the area and processed using a DC load flow model of the area. The processed measurements do not respond to line trips or power redispatches outside the area. The method extends previous methods that locate line trips in an entire network [5] so that they work in a particular area.) More generally, the border measurements can be used to effectively decouple the area from the rest of the interconnection [7]. These methods will be particularly useful when utilities or ISOs in large interconnections restrict their attention to network models and phasor measurements for only their own area.

\section{STRESS MONITORING WITH ANGLES AND POWERS}

\section{A. Quantities for stress monitoring}

Each line in the area has a limit on its real power flow that corresponds to the line thermal limit or is a proxy for other system limits. As the generation and load increase, there is increased stress on the transmission system, and lines may approach or reach their limits, especially under contingency conditions in which a line outages.

Our goal is to monitor a single quantity for the area that summarizes or captures well enough the degree to which the lines in the area are near their thermal limits. The single quantities that we consider are the real power into the area $P_{a}^{\text {into }}$ and the area angle $\theta_{\text {area }}$. The real power into the area $P_{a}^{\text {into }}$ is the sum of the real powers flowing into the area along the tie lines connected to the border buses $M_{a}$. (In practical power systems, flows in tie lines, or groups of tie lines, are monitored, and $P_{a}^{\text {into }}$ is the corresponding combined flow for the tie lines connected to the " $a$ " border buses of an area.) Ideally, the monitored quantity changes from its base case value if a line outages, and the amount of change should indicate the severity of the outage in some sense. (It turns out that $\theta_{\text {area }}$ is generally much more responsive to line outages than $P_{a}^{\text {into }}$.)

To determine the limits on the monitored quantity, we stress the power system by assuming a particular pattern of load and generation increase that increases the power flowing through the area. This stress is increased until the first line in the area reaches its thermal limit. The value of the monitored quantity in this stressed condition is its limiting value. For example, the limiting value of $P_{a}^{\text {into }}$ is written as $P_{a}^{\text {intomax }}$.

This limiting value of a monitored quantity can be determined either in the base case or in the contingency condition in which a particular line is outaged. Limits on the power flowing through the area have significant economic consequences when the limit is reached. Therefore we rank outage severity according to the corresponding limiting value of $P_{a}^{\text {intomax }}$. It is of interest to find out how much monitoring $\theta_{\text {area }}$ gives some indication of the outage severity.

\section{B. Formulas for voltage angle and power through the area}

We summarize from [1] formulas related to the area angle and power entering the area. We consider an area $\mathrm{R}$ of the 
power system with border buses $M$ and interior buses $N$. (The interior buses $N$ have no incident lines joining them to buses outside R.) The susceptance matrix from the base case DC power flow is written as $B$ or $B^{(0)}$, with subscripts indicating submatrices or elements of $B$. The following notation is used for column vectors of voltage angles and powers:

$\theta_{m} \quad$ voltage angles at border buses $M$

$P_{m} \quad$ power injected at border buses $M$

$\theta_{n} \quad$ voltage angles at interior buses $N$

$P_{n} \quad$ power injected at interior buses $N$

The vector of powers entering $\mathrm{R}$ into border buses $M$ along tie lines not in $\mathrm{R}$ is

$$
P_{m}^{\text {into }}=\sum_{j \notin \mathrm{R}}\left(-B_{m j}\right)\left(\theta_{j}-\theta_{m}\right)
$$

The vector of powers $P_{m}^{\mathrm{R}}$ entering the border buses of $\mathrm{R}$ is the sum of the power $P_{m}$ injected directly at the border buses and the power $P_{m}^{\text {into }}$ flowing into the area along the tie lines:

$$
P_{m}^{\mathrm{R}}=P_{m}+P_{m}^{\text {into }} \text {. }
$$

The susceptance matrix of the area $R$, considered as an isolated area without its tie lines, is $B_{m m}^{\mathrm{R}}$. Retaining the border buses $M$ and applying to $R$ a standard Ward or Kron reduction to eliminate the interior buses $N$, we get

$$
\begin{aligned}
& P_{m}^{\text {Rred }}=P_{m}^{\mathrm{R}}-B_{m n} B_{n n}^{-1} P_{n}, \\
& B_{m m}^{\text {Rred }}=B_{m m}^{\mathrm{R}}-B_{m n} B_{n n}^{-1} B_{n m} .
\end{aligned}
$$

The reduced subnetwork Rred is electrically equivalent to $R$ from the perspective of the border buses. Ohm's law is valid:

$$
P_{m}^{\text {Rred }}=B_{m m}^{\text {Rred }} \theta_{m} .
$$

We indicate the partition of the border buses into two sets $M_{a}$ and $M_{b}$ by specifying the row vector

$$
\left(\sigma_{a}\right)_{i}= \begin{cases}1 & \text { bus } i \text { in } M_{a} \\ 0 & \text { otherwise. }\end{cases}
$$

$\sigma_{a}$ corresponds to a new process of contracting the nodes of $M_{a}$ as explained in [1].

Now we can define our main quantities in terms of (1)-(4) and (6). The power into the area through $M_{a}$ is

$$
P_{a}^{\text {into }}=\sigma_{a} P_{m}^{\text {into }} .
$$

The susceptance of the area $b_{\text {area }}$ is

$$
b_{\text {area }}=\sigma_{a} B_{m m}^{\mathrm{Rred}} \sigma_{a}^{T} .
$$

The area angle $\theta_{\text {area }}$ is

$$
\theta_{\text {area }}=\frac{\sigma_{a} B_{m m}^{\mathrm{Rred}} \theta_{m}}{b_{a b}} .
$$

The equivalent power that flows from $M_{a}$ to $M_{b}$ through $\mathrm{R}$ is

$$
P_{\text {area }}=\sigma_{a} P_{m}^{\text {Rred }} \text {. }
$$

Ohm's law remains valid throughout the reduction and contraction so that

$$
P_{\text {area }}=b_{\text {area }} \theta_{\text {area }} .
$$

\section{Problem setup}

Now we use formulas (7)-(9), in the base case and just after each outage to determine area angle, area susceptance, and the power entering into the area. Furthermore, after we estimate the extra power that can enter into the area in the base case and after each outage, we can define the maximum amount of the area voltage angle and the maximum power entering into the area that are possible without violating any line limits.

We consider both the base case and single, non-islanding outages inside the area. These outages will cause the susceptance of the area and the area angle to change. For a general area that has parallel paths around the area that are parallel to the power flow through the area, an outage inside the area will cause some change in the power into the area tie lines. But if there are no such parallel paths around the area, as is the case for a cutset area, the power in the tie lines does not change for a non-islanding outage, and the power entering into the area will remain constant. (Note that a line outage that is non-islanding implies that all generation and load continues to be connected to all of the grid.)

In the next step, to determine the maximum area voltage angle or limit area voltage angle in the base case and after each outage, we stress the system until the first line violates its limit power flow which is the maximum power flow on that line. To do so, we determine the power transfer distribution factor for all lines considering the specified set of buses as injection buses. Then, for each line, considering its maximum amount of the power flow, the maximum value of injection which satisfies the power flow on that line can be estimated and then the minimum of these injection for all lines are considered as the maximum amount of the possible injection or stress that system can withstand in the base case or in that particular contingency before violating the limit power flow in lines. For this injection, the limit area voltage angle and limit power entering to the area can also be computed.

We use the following notation:

$P \quad$ vector of net active power injected at buses

$P_{\text {linek }} \quad$ power flow through line $k$

$P_{\text {line }} \quad$ vector of power flows through lines

$P_{\text {area }} \quad$ equivalent power flow through area

$P_{\text {stress }} \quad$ amount of power injected to stress the system

$\theta_{j} \quad$ voltage angle at bus $j$

$\theta \quad$ vector of voltage angles at buses

$\theta_{\text {area }} \quad$ voltage angle across the area

$\theta_{\text {line }} \quad$ voltage angle in each line

$B \quad$ susceptance matrix

$b_{\text {area }} \quad$ area susceptance

$\Lambda \quad$ diagonal matrix of line susceptances

$A \quad$ bus line incidence matrix

$\rho_{k}^{r s} \quad$ power transfer distribution factor for line $k$ with respect to injections in buses $r$ and $s$

It is convenient to evaluate some of the variables above in different cases and notate this as follows: 
$X \quad$ generic variable

$X^{(i)} \quad X$ evaluated for contingency number $i$.

The base case is contingency number 0 .

$X^{k \max } \quad X$ evaluated at the maximum stressed case obtained by applying stress until line $k$ reaches its maximum power flow rating.

$X^{(i) \max } \quad X$ evaluated for the maximum stressed case obtained under contingency number $i$

$X^{\text {limit }} \quad$ operating limit established for $X$

Line outage $i$ is the $i$ th outage inside the area that does not island the area. $i=0$ indicates the base case. The following calculation is done assuming the outage of line $i$, or the base case if $i=0$.

From the DC load flow with line $i$ outaged, we have

$$
P^{(i)}=B^{(i)} \cdot \theta^{(i)}
$$

where $P^{(i)}$ is the net active power injected at buses, $B^{(i)}$ is the susceptance matrix and $\theta^{(i)}$ is the bus voltage angles, all of them assuming the base case power injections. The area susceptance $b_{\text {area }}^{(i)}$ after line outage $i$ is computed from $B^{(i)}$ similarly to the computation of $b_{\text {area }}$ from $B$ in (4) and (8).

Based on (9), the area angle after each outage is computed using

$$
\theta_{\text {area }}^{(i)}=\frac{\sigma_{a} \cdot B_{e q} \cdot \theta_{m}^{(i)}}{b_{\text {area }}}
$$

Note that (13) uses the susceptance matrix and area susceptance evaluated before the outage of line $i$.

Now we determine how much more power can enter into $\mathrm{R}$ when the area is stressed until a line limit is reached. It is convenient to first consider an area stress caused by injecting power at bus $r$ (in the "generating side" outside or on the border of the area) and decreasing power at bus $s$ (in the "load side" outside or on the border of the area). The voltage angles across the lines are

$$
\theta_{\text {line }}^{(i)}=A^{T} \cdot \theta^{(i)},
$$

and the power flows in lines are

$$
P_{\text {line }}^{(i)}=\Lambda^{(i)} \cdot \theta_{\text {line }}^{(i)},
$$

where $\Lambda^{(i)}$ is the diagonal matrix of the susceptances with line $i$ outaged. The maximum amount of the increase in the power flow of line $k$ until its limit is reached is

$$
\Delta P_{\text {linek }}^{(i)}=P_{\text {linek }}^{\text {limit }}-P_{\text {linek }}^{(i)},
$$

where $P_{\text {line } k}^{\text {limit }}$ is the power flow limit of line $k$. Suppose that line $i$ is outaged and that line $k$ joins bus $u$ to bus $v$. Then the power transfer distribution factor for line $k$ is the amount of the increase in the power flow in line $k$ due to a unit injection of power in bus $r$ and a unit decrement of power in bus $s$ :

$$
\begin{aligned}
\rho_{k}^{r s(i)} & =e_{k}^{T} \Lambda^{(i)} A^{T}\left(B^{(i)}\right)^{-1}\left(e_{r}-e_{s}\right) \\
& \left.=b_{k}\left(e_{u}^{T}-e_{v}^{T}\right)\left(B^{(i)}\right)^{-1}\right)\left(e_{r}-e_{s}\right)
\end{aligned}
$$

Here $e_{r}$ denotes a vector with 1 at entry $r$ and all other entries zero. Now, the maximum amount of injection in bus $r$ and decrement from $s$ until line $k$ reaches its line limit is

$$
\Delta P^{r s(i) k \max }=\frac{\Delta P_{k}^{\operatorname{limit}(i)}}{\rho_{k}^{r s(i)}}
$$

Then the maximum possible injection $P_{\text {stress }}^{(i)}$ at the $r$ and $s$ buses which satisfies all the line limits is the minimum amount of the maximum stress for each line:

$$
P_{\text {stress }}^{(i)}=\operatorname{Min}\left\{\Delta \mathrm{P}^{\mathrm{rs}(\mathrm{i}) 1 \max }, \Delta \mathrm{P}^{\mathrm{rs}(\mathrm{i}) 2 \max }, \ldots, \Delta \mathrm{P}^{\mathrm{rs}(\mathrm{i}) \mathrm{nmax}}\right\},
$$

where $n$ is the total number of lines inside the area. Adding the amount of injection $\pm P_{\text {stress }}^{(i)}$ to the power injected at buses $r$ and $s$, the voltage angle $\theta^{(i) \max }$ corresponding to the maximum stress for the outage $i$, can be calculated from (12). Then the border bus components of $\theta^{(i) \max }$ can be extracted and written as $\theta_{m}^{(i) \max }$.

Using (1) and (7), the maximum power $P_{a}^{\text {into(i)max }}$ entering into the area corresponding to the maximum stress for the outage $i$, can be calculated as well:

$$
P_{a}^{\text {into(i)max }}=\sigma_{A} \sum_{j \in \bar{r}}\left(-B_{m j}\right)\left(\theta_{j}^{(i) \max }-\theta_{m}^{(i) \max }\right)
$$

The maximum area angle $\theta_{\text {area }}^{(i) \max }$ corresponding to the maximum stress case for the outage $i$, is a weighted combination of the border bus angles at the maximum stress case:

$$
\theta_{\text {area }}^{(\mathrm{i}) \max }=\frac{\sigma_{a} \cdot B_{e q} \cdot \theta_{m}^{(i) \max }}{b_{\text {area }}}
$$

Furthermore, the calculation given above for area stress caused by injections at bus $r$ and $s$ can be extended to more general patterns of stress that distribute the injections with specific weights among two groups of buses outside or at the border of the area.

\section{RESULTS}

The first test area for a 30 generator model of the eastern Japan is shown with the dark colored buses in Figure 2. The north-east border buses of the area are shown in red and the south-west border buses are shown in blue. This area was selected based on the position of the major generation and load in the network so that the transfer of power through the area captured a major power transfer of the system.

Using the base case DC load flow, the formula (9) for the area angle as a weighted combination of the border bus angles for this system evaluates to

$$
\begin{aligned}
\theta_{\text {area }}= & 0.0010 \theta_{45}+0.2424 \theta_{54}+0.1631 \theta_{60} \\
+ & 0.1192 \theta_{59}+0.0793 \theta_{72}+0.3494 \theta_{73}+0.0464 \theta_{39} \\
& -0.2359 \theta_{77}-0.5898 \theta_{71}-0.1741 \theta_{78}
\end{aligned}
$$

The system data does not include line limits, so we obtained artificial line limits by coordinating them so that the $\mathrm{N}-1$ criterion was minimally satisfied and then increasing each line limit by $20 \%$.

We assume the system stress to be the pattern of power injection at each border bus of the area that is proportional to the base case tie line flow for each border bus. 


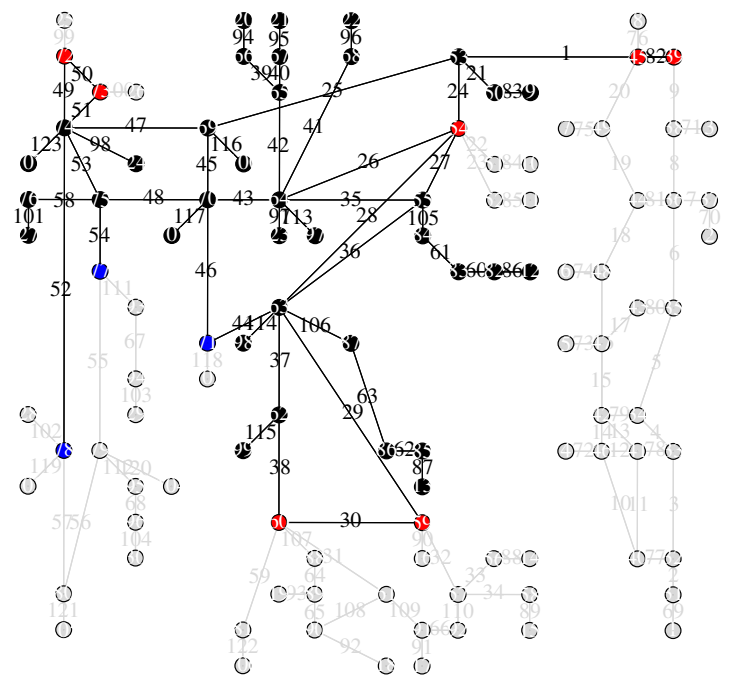

Fig. 2. First test area of 30 generator Japanese system. Buses inside the area are black, east-north border buses are red, and south-west border buses are blue.

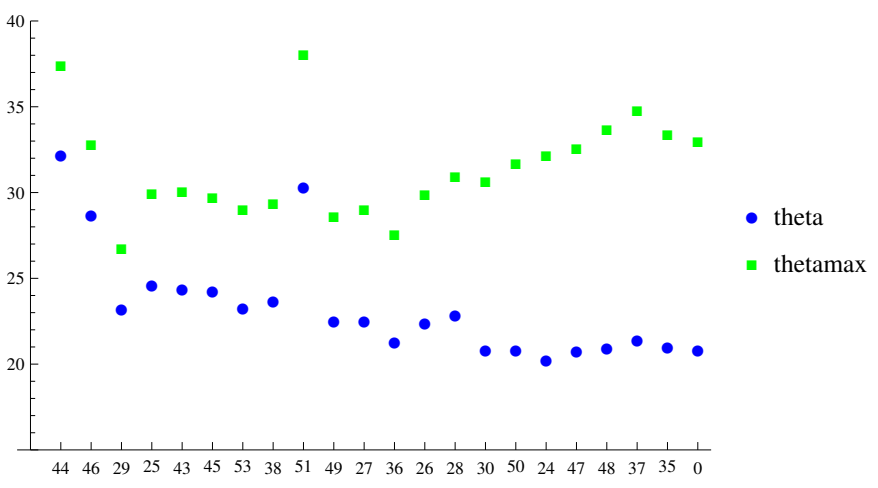

Fig. 3. Area angle $\theta_{\text {area }}^{(i)}$ and the maximum area angle $\theta_{\text {area }}^{(i) \max }$ after line outage $i$ as $i$ varies for the first test area.

The results in Figs. 3 and 4 show the area angle $\theta_{\text {area }}$ and other quantities for all the non-islanding line outages inside the area. The base case is indicated by line number 0 . The results are ordered by decreasing severity of line outage, and this can be verified by noting that the value of the maximum power $P_{a}^{\text {into }}$ that can enter the area increases from left to right. It can be seen from Figs. 3 and 4 that the area angle $\theta_{\text {area }}$ in most case decreases from left to right and so mostly responds to the severity of the outage. Since all the line outages are non-islanding and there are no parallel paths for power to flow around the area, the power $P_{a}^{\text {into }}$ entering the area is constant and is not shown in the Figs. 3 and 4. That is, in this case monitoring $P_{a}^{\text {into }}$ gives no indication of the area stress changing when one of the line outages occurs.

The area angle only depends on the line susceptance and the base case power flows. Very roughly and imprecisely speaking, it seems that the area angle changes when the line outages because the susceptance of the area changes whereas

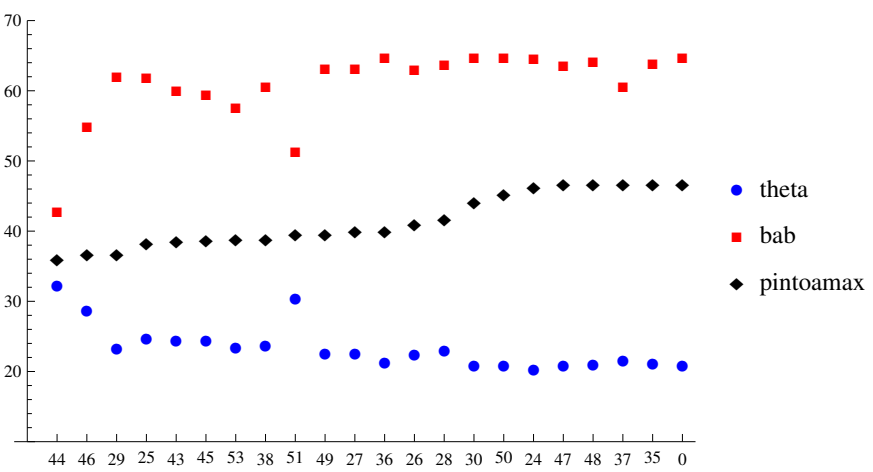

Fig. 4. Area angle $\theta_{\text {area }}^{(i)}$, area susceptance $b_{\text {area }}^{(i)}$, and maximum power entering into the area $P_{a}^{\text {into(i)max }}$ after each line outage $i$ as $i$ varies for the first test area.

the power flow entering into the area remains constant. The results show that $\theta_{\text {area }}^{(i)}$ is approximately inversely related to the area susceptance $b_{\text {area }}^{(i)}$, and this effect seems to correspond to some approximated version of Ohm's law (11).

There are some exceptions to the overall pattern of behavior such as the outages of lines 29 and 51. The outage of line 51 causes a disproportionately large decrease of the area admittance, and hence a disproportionately large increase in the area angle. This arises from the special configuration in which line 49 has a low admittance and line 51 has a high admittance. When line 51 outages, a high admittance path from border bus 73 to the area is lost and the admittance of the area sharply increases. In the case of the outage of line 29 we can see that outage severity and area susceptance don't track each other. It seems that this effect can be traced to the load at bus 99. The load at bus 99 causes line 37 to have a smaller line limit and so in the case of outage of line 29, line 37 congests at an unexpectedly low stress level.

It should be noted that the area angle does not depend on the line limits. However, the maximum area angle and the severity of the outage measured by the maximum power into the area both depend on the line limits. Thus the assumed line limits do affect the outage severity and thus the extent to which the area angle indicates outage severity. We note the limitations of the simple scheme used to coordinate and obtain the artificial line limits used in our test case. Our experience so far on another test system is that more realistic and coordinated line limits can significantly improve the results.

The angle monitoring can work to some extent for an arbitrarily chosen area, but choosing a better area can give area angles that better summarize the effect of line outages as we now discuss.

One method to choose a good area is to select the $M_{a}$ border near large generation and the $M_{b}$ border near load in the network. Then the area angle from $M_{a}$ to $M_{b}$ reflects dominant power flows in the network and we get a better indicator for outages inside the area. We used this principle to choose the first test area of Fig. 2.

In the first test area, border bus 54 excludes two generators from the area. Removing border bus 54 from the first test area yields a second test area that includes the two generators inside the area. The results for the second test area are shown in Fig. 
5 , and it can be seen that area angle still responds, but tracks the severity of the line outage more imperfectly.

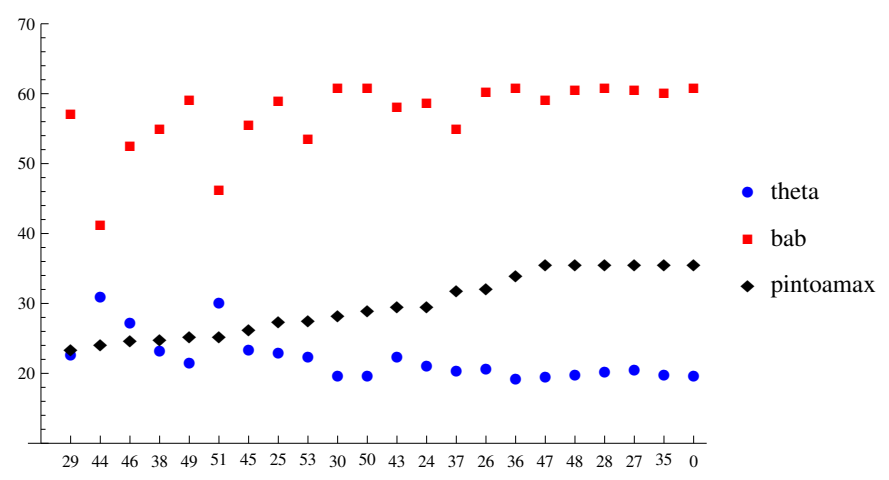

Fig. 5. Area angle $\theta_{\text {area }}^{(i)}$, area susceptance $b_{\text {area }}^{(i)}$, and maximum power entering into the area $P_{a}^{\text {into(i)max }}$ after each line outage $i$ for the second test area.

It seems better to avoid large load or generation inside the area. The anomaly of line outage 29 in figure 2 was attributed to load inside the area in the previous discussion. Another example is the third test area shown with the loads inside the area in Fig. 6. The resulting area angles are shown in Fig. 7. For the outage of lines 37 and 38 we get the same susceptance for the area, but the maximum power that can enter the area after the outage, which indicates the severity of the outage, is much greater for line 38 than for line 37 . The difference in severity seems to be related to the load at bus 99 . The third test area also includes a small part of the network, namely bus 73 and generator bus 26 and line 100, which is not in the main power flow from $M_{a}$ to $M_{b}$ when either line 50 or line 51 is outaged. The outage of either line 50 or line 51 has a significant effect on the area admittance but little impact on the severity.

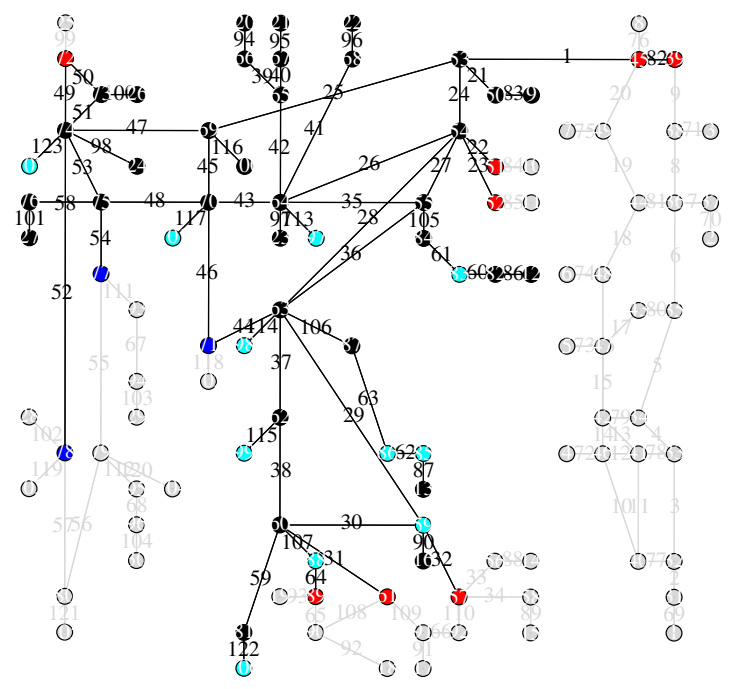

Fig. 6. Third test area. Loads inside the area are shown in cyan.

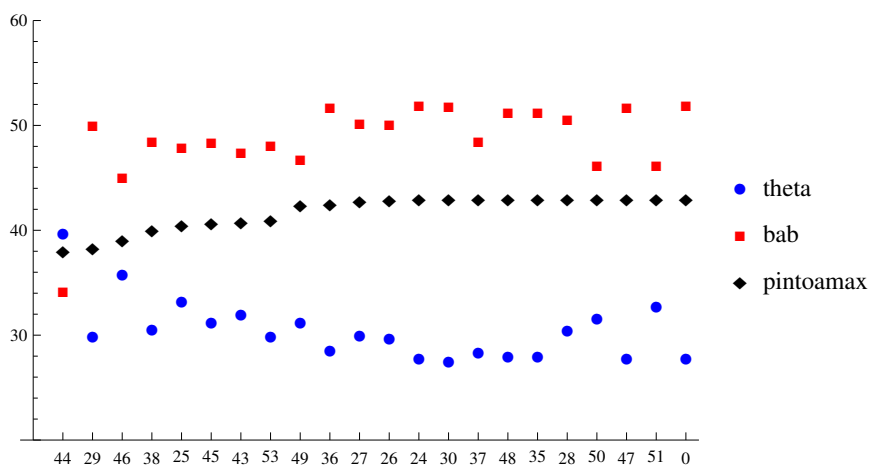

Fig. 7. Area angle $\theta_{\text {area }}^{(i)}$, area susceptance $b_{\text {area }}^{(i)}$, and maximum power entering into the area $P_{a}^{\text {into(i)max }}$ after each line outage $i$ for the third test area.

\section{CONCLUSION}

We explore monitoring of area stress due to non-islanding line outages with area angles in a 30-bus Japanese test system. The area angle is easy to compute from synchrophasor measurements at the border buses of the area and it satisfies circuit laws. The area angle responds to the line outages by increasing. Given a suitable choice of area that separates the main generation and load, the amount of the increase in the area angle approximates the outage severity in most cases. In contrast, the power entering the area does not indicate these line outages. These first results suggest that real-time monitoring of angles across areas could be a promising way to help operators quickly detect stress due to line outages. Issues to be resolved in future work include further guidelines for good choices of area, the effects of multiple and islanding outages, setting actionable thresholds to distinguish the severe outages, and the possible use of multiple angles across an area.

\section{ACKNOWLEDGMENTS}

$\mathrm{AD}$ and ID gratefully acknowledge funding in part from Fuji Electric Co. Ltd. and NSF grant CPS-1135825. This paper was prepared with the support in part of the U.S. Department of Energy for "The Future Grid to Enable Sustainable Energy Systems," an initiative of the Power Systems Engineering Research Center.

\section{REFERENCES}

[1] I. Dobson, Voltages across an area of a network, IEEE Transactions on Power Systems, vol. 27, no. 2, May 2012, pp. 993-1002.

[2] I. Dobson, M. Parashar, C. Carter, Combining phasor measurements to monitor cutset angles, Forty-third Hawaii International Conference on System Sciences, Kauai, Hawaii, January 2010.

[3] I. Dobson, M. Parashar, A cutset area concept for phasor monitoring, IEEE PES General Meeting, Minneapolis, MN USA, July 2010.

[4] I. Dobson, New angles for monitoring areas, IREP Symposium, Bulk Power System Dynamics and Control - VIII, Buzios, Brazil, Aug. 2010.

[5] J.E. Tate, T.J. Overbye, Line outage detection using phasor angle measurements, IEEE Trans. Power Syst., vol 23, no 4, Nov 2008, pp. 1644-1652.

[6] H. Sehwail, I. Dobson, Locating line outages in a specific area of a power system with synchrophasors, North American Power Symposium (NAPS), Urbana-Champaign IL, Sept. 2012.

[7] H.Sehwail, I.Dobson, Applying synchrophasor computations to a specific area, IEEE Trans. Power Systems, vol 28, no 3, Aug 2013, pp. 3503-3504.

[8] G.J. Lopez, J.W. Gonzalez, R.A. Leon, H.M. Sanchez, I.A. Isaac, H.A. Cardona, Proposals based on cutset area and cutset angles and possibilities for PMU deployment, IEEE PES General Meeting, San Diego CA, July 2012. 